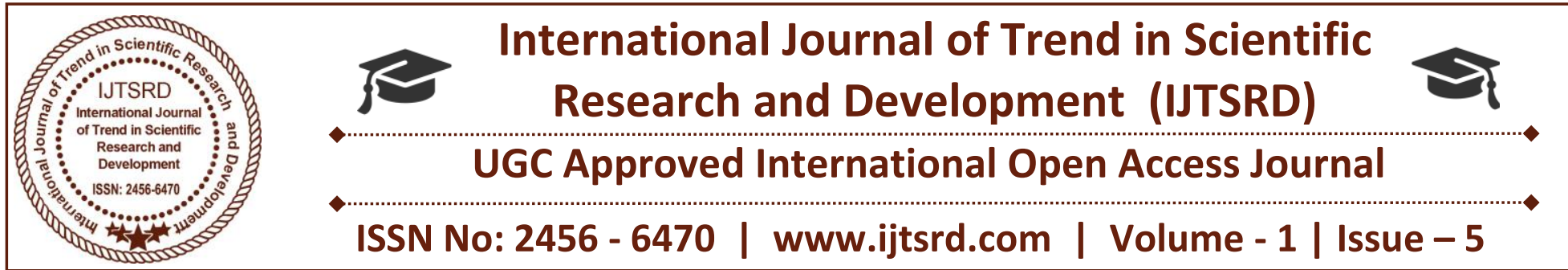

\title{
Strong Regular L-Fuzzy Graphs
}

\section{R. Seethalakshmi}

Saiva Bhanu Kshatriya College, Aruppukottai, Tamilnadu, India

\section{R.B. Gnanajothi}

V.V.Vanniaperumal College For Women, Virudhunagar, Tamilnadu, India

\section{ABSTRACT}

In this paper, the notion of regular and strong regular L-fuzzy graph is introduced. The conditions for strong regularity of L-fuzzy graphs on cycle and star graph are derived.

Keywords: L-fuzzy graph, strong L-fuzzy graph, regular L-fuzzy graph, strong regular L-fuzzy graph

AMS Classification: 05C72, 03E72

\section{INTRODUCTION}

The first definition of fuzzy graph was proposed by Kaufmann [3] using fuzzy relations introduced by Zadeh[9]. The theory of fuzzy graphs was introduced by Azriel Rosenfeld [7] in 1975. Generalizing the notion of fuzzy sets, L. Goguen [2] introduced the notion of L-fuzzy sets. Pramada Ramachandran and Thomas [6] introduced the notion of L-fuzzy graphs and the degree of a vertex in a L-fuzzy graph. This motivated us to introduce the notion of regular L-fuzzy graphs and strong regular L-fuzzy graphs. Some preliminary, but primary work has been carried out. Comparison of set magic graphs and L-fuzzy structure introduces a new platform to work on fuzzy graph.

\section{PRELIMINARIES}

Definition 2.1: [4] A fuzzy graph $G=(\sigma, \mu)$ is a pair of functions $\sigma: V \rightarrow[0,1]$ and $\mu: V X V \rightarrow[0,1]$ with $\mu(u, v) \leq \sigma(u) \wedge \sigma(v), \forall u, v \in V$, where $\mathrm{V}$ is a finite nonempty set and $\wedge$ denote minimum.

Definition 2.2: [4] The graph $G^{*}=(V, E)$ is called the underlying crisp graph of the fuzzy graph $\mathrm{G}$, where $V=\{u / \sigma(u) \neq o\}$ and $E=\{(u, v) \in V X V / \mu(u, v) \neq 0\}$.

Definition 2.3: [5] A fuzzy graph $G=(\sigma, \mu)$ is defined to be a strong fuzzy graph if

$$
\mu(u, v)=\sigma(u) \wedge \sigma(v), \forall(u, v) \in E .
$$


International Journal of Trend in Scientific Research and Development (IJTSRD) ISSN: 2456-6470

Definition 2.4: [5] A fuzzy graph $G=(\sigma, \mu)$ is defined to be a complete fuzzy graph if

$$
\mu(u, v)=\sigma(u) \wedge \sigma(v), \forall u, v \in V .
$$

Definition 2.5: [5] Let $G=(\sigma, \mu)$ be a fuzzy graph on $G^{*}=(V, E)$. The fuzzy degree of a node $u \in V$ is defined as $(f d)(u)=\sum_{u \neq v, v \in V} \mu(u, v)$. G is said to be regular fuzzy graph if each vertex has same fuzzy degree. If $(f d)(v)=k, \forall v \in V$, then $\mathrm{G}$ is said to be $k$-regular fuzzy graph.

Definition 2.6: [6] Let $(L, \vee, \wedge)$ be a complete lattice. A nonempty set $\mathrm{V}$ together with a pair of functions $\sigma$ : $V \rightarrow L$ and $\mu: V X V \rightarrow L$ with $\mu(u, v) \leq \sigma(u) \wedge \sigma(v), \forall u, v \in V$, is called an L-fuzzy graph. It is denoted by $G_{L}=(V, \sigma, \mu)$.

Definition 2.7: [6] Let $G_{L}=(V, \sigma, \mu)$ be an L-fuzzy graph. The L-fuzzy degree $d_{L}(u)$ of a vertex $u$ in $G_{L}$ is defined as $d_{L}(u)=V_{u \in V, u \neq v} \mu(u, v)$.

Definition 2.8 : [1] A graph $\mathrm{G}=(\mathrm{V}, \mathrm{E})$ is said to have a set-magic labeling if its edges can be assigned distinct subsets of a set $\mathrm{X}$ such that for every vertex $\mathrm{u}$ of $\mathrm{G}$, the union of the subsets assigned to the edges incident at $\mathrm{u}$ is $X$.

A graph is said to be a set-magic graph if it admits a set-magic labeling.

\section{REGULAR L-FUZZY GRAPH}

The above definition of degree in an L-fuzzy graph does not coincide with usual definition of degree in a fuzzy graph. Any fuzzy graph can be treated as an L-fuzzy graph taking the lattice $([0,1], \leq)$. Fuzzy degree of a vertex $u$, denoted as $f_{d}(u)$, defined as the sum of $\mu(u, v)$, where $u \in V, u \neq v$.

For further discussion, L-fuzzy degree will be taken into account.

Definition 3.1: Let $G_{L}=(V, \sigma, \mu)$ be an L-fuzzy graph. $G_{L}$ is said to be a regular L-fuzzy graph if each vertex has the same L-fuzzy degree. If $d_{L}(v)=k, \forall v \in V$, for some $k \in L$, then $G_{L}$ is called a $k$ - regular L-fuzzy graph.

Example 3.2 : Let $S=\{a, b, c\}$ and $\mathrm{L}=\wp(S)$, the power set of $S$.

Then $(L, \subseteq)$ is a complete lattice. Let $V=\left\{v_{1}, v_{2}, v_{3}, v_{4}, v_{5}\right\}$

Define $\sigma: V \rightarrow L$ and $\mu: V X V \rightarrow L$ by $\sigma\left(v_{1}\right)=\{a\}, \sigma\left(v_{2}\right)=\{a, b\}=\sigma\left(v_{4}\right)$,

$\sigma\left(v_{3}\right)=\sigma\left(v_{5}\right)=\{a, c\}$ and

$$
\mu\left(v_{1}, v_{2}\right)=\mu\left(v_{1}, v_{3}\right)=\mu\left(v_{1}, v_{4}\right)=\mu\left(v_{2}, v_{3}\right)=\mu\left(v_{3}, v_{5}\right)=\mu\left(v_{3}, v_{4}\right)=\{a\}
$$

Then $d_{L}(u)=v_{v \in V, u \neq v} \mu(u, v)=\{a\}, \forall u \in V$. Then $G_{L}=(V, \sigma, \mu)$ is a regular L-fuzzy graph.

Example 3.3 : Consider the set $S$ and the complete lattice $L=\wp(S)$ given as in Example 3.2.

Let $V=\left\{v_{1}, v_{2}, v_{3}\right\}$ 
Define $\sigma: V \rightarrow L$ and $\mu: V X V \rightarrow L$ by $\sigma\left(v_{1}\right)=\{a, b, c\}, \sigma\left(v_{2}\right)=\{a, b\}, \sigma\left(v_{3}\right)=\{a, b\}$ and $\mu\left(v_{1}, v_{2}\right)=\{b\}$, $\mu\left(v_{2}, v_{3}\right)=\{a, b\}, \mu\left(v_{3}, v_{1}\right)=\{a\}$. Then $G_{L}=(V, \sigma, \mu)$ is a L-fuzzy graph.

Example 3.4 : Consider the set $\mathrm{S}$ and $\wp(S)$ as given in example 3.2.

Let $V=\left\{v_{1}, v_{2}, v_{3}, v_{4}, v_{5}\right\}$

Define $\sigma: V \rightarrow L$ and $\mu: V X V \rightarrow L$ by

$\sigma\left(v_{1}\right)=\{a\}, \sigma\left(v_{2}\right)=\{a, b\}, \sigma\left(v_{3}\right)=\{a, c\}, \sigma\left(v_{4}\right)=\{a, b, c\}, \sigma\left(v_{5}\right)=\{b, c\}$ and

$$
\mu\left(v_{1}, v_{2}\right)=\mu\left(v_{2}, v_{3}\right)=\mu\left(v_{1}, v_{3}\right)=\mu\left(v_{1}, v_{4}\right)=\{a\}
$$

$\mu\left(v_{3}, v_{5}\right)=\{c\}, \mu\left(v_{3}, v_{4}\right)=\{a, c\}, \mu\left(v_{4}, v_{5}\right)=\{b, c\}$

Then $G_{L}=(V, \sigma, \mu)$ is a non-regular L-fuzzy graph.

Remark : In general, an L-fuzzy graph, $G_{L}=(V, \sigma, \mu)$, where $\mu$ is a constant function, is a regular L-fuzzy graph.

Theorem 3.5 : Any set magic graph admits a regular L-fuzzy graph structure.

Proof : Let $\mathrm{G}=(\mathrm{V}, \mathrm{E})$ be a set magic graph. Then $\mathrm{G}$ admits a set magic labeling $l$.

That is, the edges of $\mathrm{G}$ can be assigned distinct subsets of a set $\mathrm{X}$ such that for every vertex $u$ of $\mathrm{G}$, union of subsets assigned to the edges incident at $u$ is $\mathrm{X}$.

Let $\mathrm{L}=\wp(X)$, the power set of $X$. Then $(L, \subseteq)$ is a complete lattice.

Define $\sigma: V \rightarrow L$ and $\mu: E \rightarrow L$ as follows:

$\sigma(v)=X, \forall v \in V$ and $\mu(e)=l(e)$, for all edges $e$ of $\mathrm{G}$ and $l(e) \in L$.

Since, $l(e) \subseteq X, \mu(e) \leq \sigma(u) \wedge \sigma(v)$, for all $e=(u, v) \in E$.

Then, $G_{L}=(V, \sigma, \mu)$ is an L-fuzzy graph.

For all $\mathrm{u} \in V, \quad d_{L}(u)=\mathrm{V}_{v \in V, v \neq u} \mu(u, v)$

$$
\begin{aligned}
& =\mathrm{V}_{e=(u, v), v \neq u} \mu(e) \\
& =\mathrm{V}_{e=(u, v), v \neq u} l(e) \\
& =\mathrm{X}, \text { (since } l \text { is a set magic labeling) }
\end{aligned}
$$

Hence, $G_{L}$ is a regular L-fuzzy graph. 


\section{STRONG REGULAR L-FUZZY GRAPH}

Definition 4.1 : An L-fuzzy graph $G_{L}=(\mathrm{V}, \sigma, \mu)$ is said to be a strong regular L-fuzzy graph if $\mu(u, v)=\sigma(u) \wedge$ $\sigma(v)$, for all edges $(\mathrm{u}, \mathrm{v})$ of $G_{L}$ and the L-fuzzy degree $d_{L}(v)$ is constant, for all $v \in V$.

Example 4.2 : Let $S=\{a, b, c\}$ and $\mathrm{L}=\wp(S)$, the power set of $S$. Then $(L, \subseteq)$ is a complete lattice. Let $V=$ $\left\{v_{1}, v_{2}, v_{3}, v_{4}\right\}$

Define $\sigma: V \rightarrow L$ by $\sigma\left(v_{1}\right)=\sigma\left(v_{3}\right)=\{a, b, c\}, \sigma\left(v_{2}\right)=\sigma\left(v_{4}\right)=\{a, b\}$ and

$$
\mu\left(v_{1}, v_{2}\right)=\mu\left(v_{2}, v_{3}\right)=\mu\left(v_{3}, v_{4}\right)=\mu\left(v_{4}, v_{1}\right)=\{a, b, c\}
$$

Then $G_{L}=(V, \sigma, \mu)$ is a strong regular L-fuzzy graph.

Example 4.3 : If $\sigma$ and $\mu$ are constants in a L-fuzzy graph $G_{L}$, then $G_{L}$ is strong regular L-fuzzy graph.

Remark : If $G_{L}$ is a strong regular L-fuzzy graph, then $G_{L}$ is a regular L-fuzzy graph. However, the converse need not be true, in general. This is seen from Example 3.3.

Note In [8], it has been proved that no fuzzy graph on a star graph with at least two spokes is strong regular. But in case of L-fuzzy graph, there exist a strong regular L-fuzzy structure on star graph.

Example 4.3 : Let $\mathrm{L}=\{1,2,3\} .(L, \leq)$ is a complete lattice. Let $V=\left\{v, v_{1}, v_{2}, v_{3}, v_{4}\right\}$

Define $\sigma: V \rightarrow L$ by $\sigma(v)=1, \sigma\left(v_{1}\right)=1, \sigma\left(v_{2}\right)=2, \sigma\left(v_{3}\right)=3, \sigma\left(v_{4}\right)=4$

and $\mu\left(v, v_{i}\right)=1, i=1,2,3,4$. Then, $d_{L}\left(v_{1}\right)=d_{L}\left(v_{2}\right)=d_{L}\left(v_{3}\right)=d_{L}\left(v_{4}\right)=1$.

Hence, $G_{L}=(V, \sigma, \mu)$ is a strong regular L-fuzzy graph on a star graph.

Example 4.4 :Let $S=\{a, b, \mathrm{c}\}$ and $\mathrm{L}=\wp(S)$, the power set of $S .(L, \subseteq)$ is a lattice.

1. Let $=\left\{v_{1}, v_{2}, v_{3}, v_{4}\right\}$.

Define $\sigma: V \rightarrow L$ by $\sigma\left(v_{1}\right)=\{a, b\}, \sigma\left(v_{2}\right)=\{a\}, \sigma\left(v_{3}\right)=\{a, c\}, \sigma\left(v_{4}\right)=\{a\}$ and $\mu\left(v_{1}, v_{2}\right)=\mu\left(v_{2}, v_{3}\right)=\mu\left(v_{3}, v_{4}\right)=\mu\left(v_{4}, v_{1}\right)=\{a\}$.

Then, $G_{L}=(V, \sigma, \mu)$ is strong regular L-fuzzy graph.

2. Let $=\left\{v, v_{1}, v_{2}, v_{3}, v_{4}\right\}$. Define $\sigma: V \rightarrow L$ by

$$
\sigma\left(v_{1}\right)=\{a, b, c\}, \sigma\left(v_{2}\right)=\{a\}, \sigma\left(v_{3}\right)=\{a, c\}, \sigma\left(v_{4}\right)=\{a, b\}, \sigma(v)=\{a\}
$$

and $\mu\left(v, v_{1}\right)=\mu\left(v, v_{2}\right)=\mu\left(v, v_{3}\right)=\mu\left(v, v_{4}\right)=\{a\}$.

Then, $G_{L}=(V, \sigma, \mu)$ is a strong regular L-fuzzy graph.

Theorem 4.5 Let $G_{L}=(V, \sigma, \mu)$ be the L-fuzzy graph.

1. If the underlying graph $G_{L}^{*}$ is a cycle and if $\mu$ is a constant say $\mathrm{k}$, for all edges $(\mathrm{u}, \mathrm{v})$ and $\sigma(u) \geq k$, for all $u \in V$, then $G_{L}$ is a strong regular L-fuzzy graph.

2. If the underlying graph $G_{L}^{*}$ is a star graph with $V=\left\{v, v_{1}, v_{2}, \ldots . v_{n}\right\}$ and if $\sigma(v) \leq \sigma\left(v_{i}\right), \forall i=1,2,3, \ldots . n$ and $\mu\left(v, v_{i}\right)=\sigma(v)$, for all the edges $\left(v, v_{i}\right)$, then $G_{L}$ is a strong regular L-fuzzy graph. 


\section{Proof}

1. Let $\mu=$ constant $=\mathrm{k}$ (say).

Since, $\sigma(u) \geq k$, for all $u \in V, \sigma(u) \wedge \sigma(v) \geq k=\mu(u, v)$.

From the definition of L-fuzzy graph, $\mu(u, v) \leq \sigma(u) \wedge \sigma(v)$.

Then, $\mu(u, v)=\sigma(u) \wedge \sigma(v)$.

Also, $d_{L}(u)=V_{u \in V, u \neq v} \mu(u, v)=V_{u \in V, u \neq v} k=k, \forall u \in V$.

Hence, $G_{L}$ is strong regular L-fuzzy graph.

2. Let $\sigma(v) \leq \sigma\left(v_{i}\right), \forall i=1,2,3, \ldots \ldots n$.

Therefore, $\sigma\left(v_{i}\right) \wedge \sigma(v) \leq \sigma(v)=\mu\left(v, v_{i}\right)$.

From the definition of L-fuzzy graph, $\mu\left(v, v_{i}\right) \leq \sigma\left(v_{i}\right) \wedge \sigma(v)$.

Hence, $\mu\left(v, v_{i}\right)=\sigma(v) \wedge \sigma\left(v_{i}\right), \forall i=1,2,3, \ldots \ldots n$.

Now, $d_{L}\left(v_{i}\right)=\bigvee_{v \in V} \mu\left(v_{i}, v\right), \forall i=1,2,3, \ldots \ldots$.

$$
\begin{aligned}
& =\mathrm{V} \sigma(v) \\
& =\sigma(v)
\end{aligned}
$$

and $d_{L}(v)=\vee_{v_{i} \in V} \mu\left(v, v_{i}\right), \forall i=1,2,3, \ldots \ldots n$.

$$
\begin{aligned}
& =\mathrm{V} \sigma(v) \\
& =\sigma(v)
\end{aligned}
$$

Thus, $G_{L}$ is strong regular L-fuzzy graph.

Theorem 4.6 : For any strong regular L-fuzzy graph $G_{L}=(V, \sigma, \mu)$ whose crisp graph $G_{L}^{*}$ is a path, $\mu$ is a constant function.

Proof Let $V=\left\{v_{0}, v_{1}, v_{2}, \ldots \ldots, v_{n}\right\} . E\left(G_{L}^{*}\right)=\left\{v_{0} v_{1}, v_{1} v_{2}, v_{2} v_{3}, \ldots \ldots \ldots, v_{n-1} v_{n}\right\}$

$\sigma\left(v_{i}\right)=b_{i}$, for $i=0,1,2, \ldots \ldots \ldots n$ and

$\mu\left(v_{i-1}, v_{i}\right)=a_{i}$, for $i=1,2, \ldots \ldots \ldots n$, where $a_{i}, b_{i} \in(L, \leq)$

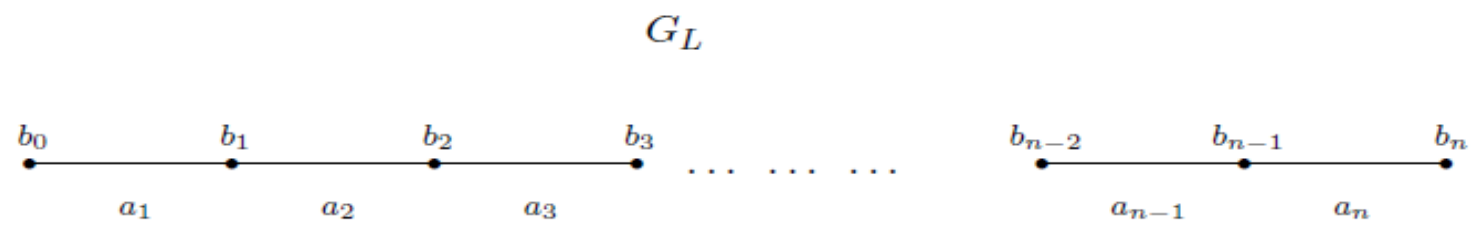

Since, $G_{L}$ is a strong regular L-fuzzy graph, we have

$a_{1}=k . a_{i} \vee a_{i+1}=k$, for $i=1,2, \ldots \ldots \ldots n-2, a_{n}=k$, where $k \in L$

$b_{i-1} \wedge b_{i}=a_{i}$, for $i=1,2, \ldots \ldots \ldots n$

(1) $\Rightarrow a_{i} \leq k, i=1,2, \ldots \ldots \ldots, n$

Also $(2) \Rightarrow a_{i} \leq b_{i-1}$ and $a_{i} \leq b_{i}, i=1,2, \ldots \ldots \ldots, n$

$\Rightarrow\left(a_{1} \leq b_{0}, a_{1} \leq b_{1}\right),\left(a_{2} \leq b_{1}, a_{2} \leq b_{2}\right), \ldots \ldots \ldots,\left(a_{n} \leq b_{n-1}, a_{n} \leq b_{n}\right)$

$\Rightarrow\left(a_{1} \leq b_{0}\right),\left(a_{1}, a_{2} \leq b_{1}\right),\left(a_{2} \leq b_{2}, a_{3} \leq b_{2}\right), \ldots \ldots \ldots,\left(a_{n-1} \leq b_{n}, a_{n} \leq b_{n}\right),\left(a_{n} \leq b_{n}\right)$ 
International Journal of Trend in Scientific Research and Development (IJTSRD) ISSN: 2456-6470

$$
\begin{aligned}
& \Rightarrow k \leq b_{0}, a_{i} \vee a_{i+1} \leq b_{i}, i=1,2, \ldots \ldots \ldots, n-1, k \leq b_{n} . \\
& \Rightarrow k \leq b_{0}, k \leq b_{i}, i=1,2, \ldots \ldots \ldots, n-1, k \leq b_{n} . \\
& \Rightarrow k \leq b_{0}, b_{i-1} \wedge b_{i}, i=1,2, \ldots \ldots \ldots, n \text {. } \\
& \Rightarrow k \leq a_{i}, \text { for } i=1,2, \ldots \ldots \ldots, n
\end{aligned}
$$

Equations (3) and (4) $\Rightarrow, a_{i}=k$, for $i=1,2, \ldots \ldots, n$.

Hence, $\mu$ is a constant function.

Theorem 4.7 : For any strong regular L-fuzzy graph $G_{L}=(V, \sigma, \mu)$ whose crisp graph $G_{L}^{*}$ is a cycle, $\mu$ is a constant function.

Proof Let $V=\left\{v_{1}, v_{2}, \ldots \ldots, v_{n}\right\} . E\left(G_{L}^{*}\right)=\left\{v_{1} v_{2}, v_{2} v_{3}, \ldots \ldots \ldots, v_{n-1} v_{n}, v_{n} v_{1}\right\}$ $\sigma\left(v_{i}\right)=b_{i}$, for $i=1,2, \ldots \ldots \ldots n$ and $\left(v_{i}, v_{i+1}\right)=a_{i}$, for $i=1,2, \ldots \ldots \ldots n$, where

$$
v_{n+1}=v_{1} \text { and } a_{i}, b_{i} \in(L, \leq)
$$

$$
G_{L}
$$

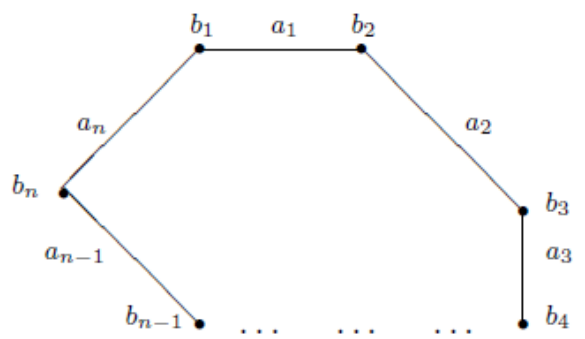

Since, $G_{L}$ is a strong regular L-fuzzy graph, we have

$a_{1}=k . a_{i} \vee a_{i+1}=k$, for $i=1,2, \ldots \ldots \ldots n-1$, where $k \in L$

$b_{i} \wedge b_{i+1}=a_{i}$, for $i=1,2, \ldots \ldots \ldots n$, since $v_{n+1}=v_{1}, b_{n+1}=b_{1}$

(1) $\Rightarrow a_{i} \leq k, i=1,2$,

Also (2) $\Rightarrow a_{i} \leq b_{i}$ and $a_{i} \leq b_{i+1}, i=1,2, \ldots \ldots \ldots, n$

$$
\begin{aligned}
& \Rightarrow\left(a_{1} \leq b_{1}, a_{1} \leq b_{2}\right),\left(a_{2} \leq b_{2}, a_{2} \leq b_{3}\right), \ldots \ldots \ldots,\left(a_{n} \leq b_{n}, a_{n} \leq b_{n+1}=b_{1}\right) \\
& \Rightarrow\left(a_{1} \leq b_{1}\right),\left(a_{1}, a_{2} \leq b_{2}\right),\left(a_{2}, a_{3} \leq b_{3}\right), \ldots \ldots \ldots,\left(a_{n-1}, a_{n} \leq b_{n}\right) \\
& \Rightarrow k \leq b_{1}, a_{i} \vee a_{i+1} \leq b_{i+1}, i=1,2, \ldots \ldots \ldots, n-1 \\
& \Rightarrow k \leq b_{1}, k \leq b_{i+1}, i=1,2, \ldots \ldots \ldots, n-1 \\
& \Rightarrow k \leq b_{i} \wedge b_{i+1}, i=1,2, \ldots \ldots \ldots, n . \\
& \Rightarrow k \leq a_{i}, \text { for } i=1,2, \ldots \ldots, n \text {-.-.-.-.-.-- }
\end{aligned}
$$


Equations (3) and (4) $\Rightarrow, a_{i}=k$, for $i=1,2, \ldots \ldots \ldots, n$.

Hence, $\mu$ is a constant function.

\section{REFERENCES}

1) Acharya B D, Set valuations of a graph and their applications, MRI Lecture Notes in Applied Mathematics, No 2, Mehta Research Intitute, Allahabad, 1983.

2) Goguen. J. A, L-Fuzzy sets, J. Math. Anal.Appl. 18, (1967), 145 - 174.

3) Kaufmann, Introduction a la theorie des sous - ensembles flous, Elements theoriques de base, Parisi Masson et cie 1976.

4) Morderson, J.N, Nair, P.S, Fuzzy Graphs and Fuzzy Hyper Graphs, Physics - verlag, Heidelberg (2000)

5) Nagoorgani, A and Chandrasekaran V.T, A First Look at Fuzzy Graph Theory(2010)

6) Pramada Ramachandran, Thomas K V, On Isomorphism of L-Fuzzy graphs, Annals of Fuzzy Mathematics and Informatics, Vol 11, No 2(February 2016), pp 301-313

7) Rosenfeld A, Fuzzygraphs In : Zadeh, L.A. Fu, K.S.Shimura, M (Eds), Fuzzy sets and their Applications (Academic Press, Newyark) pp.77-95(1975).

8) Seethalakshmi R and Gnanajothi R.B, On Strong regular fuzzy graph, International Journal of Mathematical Sciences and Engineering Applications, ISSN 0973-9424, Vol 6 No.1( January 2012),pp 161-173.

9) Zadeh L.A. Similarity relations and fuzzy ordering Information sciences 971; 3(2): 177-200. 